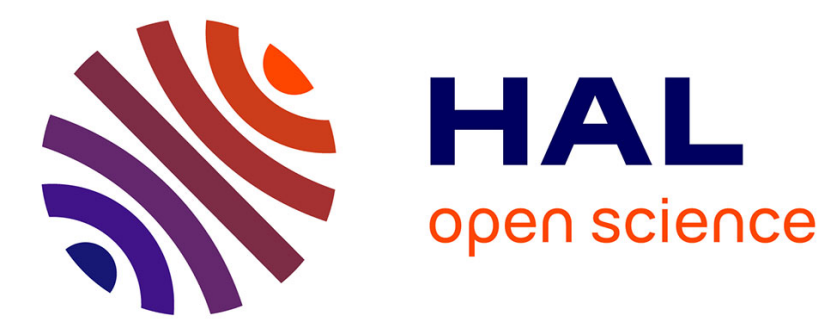

\title{
Young and rough differential inclusions
}

Ismaël Bailleul, Antoine Brault, Laure Coutin

\section{To cite this version:}

Ismaël Bailleul, Antoine Brault, Laure Coutin. Young and rough differential inclusions. Revista Matemática Iberoamericana, 2021, 37 (4), pp.1489 - 1512. 10.4171/rmi/1236 . hal-02148440v2

\section{HAL Id: hal-02148440 \\ https://hal.science/hal-02148440v2}

Submitted on 7 Sep 2020

HAL is a multi-disciplinary open access archive for the deposit and dissemination of scientific research documents, whether they are published or not. The documents may come from teaching and research institutions in France or abroad, or from public or private research centers.
L'archive ouverte pluridisciplinaire HAL, est destinée au dépôt et à la diffusion de documents scientifiques de niveau recherche, publiés ou non, émanant des établissements d'enseignement et de recherche français ou étrangers, des laboratoires publics ou privés. 


\title{
Young and rough differential inclusions
}

\author{
I. BAILLEUL, A. BRAULT and L. COUTIN
}

\begin{abstract}
We define in this work a notion of Young differential inclusion $d z_{t} \in F\left(z_{t}\right) d x_{t}$,

for an $\alpha$-Hölder control $x$, with $\alpha>1 / 2$, and give an existence result for such a differential system. As a by-product of our proof, we show that a bounded, compact-valued, $\gamma$-Hölder continuous set-valued map on the interval $[0,1]$ has a selection with finite p-variation, for $p>1 / \gamma$. We also give a notion of solution to the rough differential inclusion

$$
d z_{t} \in F\left(z_{t}\right) d t+G\left(z_{t}\right) d \mathbf{X}_{t},
$$

for an $\alpha$-Hölder rough path $\mathbf{X}$ with $\alpha \in\left(\frac{1}{3}, \frac{1}{2}\right]$, a set-valued map $F$ and a single-valued one form $G$. Then, we prove the existence of a solution to the inclusion when $F$ is bounded and lower semi-continuous with compact values, or upper semi-continuous with compact and convex values.
\end{abstract}

\section{1 - Introduction}

\section{1 - Setting}

One of the motivations for considering differential inclusions comes from the study of differential equations with discontinuous coefficients. In the setting of a possibly timedependent widely discontinuous vector field on $\mathbb{R}^{d}$, it makes sense to replace the dynamical prescription

$$
\dot{z}_{t}=f\left(t, z_{t}\right)
$$

by

$$
\dot{z}_{t} \in F\left(t, z_{t}\right) \text {, }
$$

where $F(t, z)$ is here the closed set of cluster points ot $F(s, w)$, as $(s, w)$ converges to $(t, z)$. This somehow accounts for the impossiblity to make measurements with absolute precision. It also makes sense to take for $F(t, z)$ the convex hull of the former set. A set-valued application $F$ is a map from $[0, T] \times \mathbb{R}^{d}$ into the power set of $\mathbb{R}^{d}$. Different natural setvalued extensions of $f$ may have different regularity properties; in any case, switching from the differential equation prescription to the differential inclusion formulation somehow allows to account for the uncertainty in the modeling. A Caratheodory solution of the differential inclusion

$$
\dot{z}_{t} \in F\left(t, z_{t}\right), t \in[0, T], z_{0}=\xi \in \mathbb{R}^{d}
$$

is an absolutely continuous path $z$ started from $\xi$, whose derivative $\dot{z}$ satisfies

$$
\dot{z}_{t} \in F\left(t, z_{t}\right)
$$


at almost all times $t \in[0, T]$. Existence of solutions of differential inclusions and their properties were widely studied; see for instance J.P. Aubin and A. Cellina's book [1] for an authoritative pedagogical treatment. Equation (1.1) has at least one solution under two kinds of assumptions on the set valued map $F$ : either $F$ is upper semicontinuous with compact convex values, or $F$ is bounded and lower semicontinuous with compact values. Recall that a set-valued function $F$ on $[0, T] \times \mathbb{R}^{d}$ is said to be upper semicontinuous if one can associate to every spacetime point $(s, w)$ an open neighbourhood $U$ of $(s, w)$ and an open neighbourhood $V$ of $F(s, w)$ such that $F(U) \subset V$, and $F$ is said to be lower semicontinuous if for any $(s, w)$, any $w^{\prime} \in F(s, w)$ and any neighbourhood $V\left(w^{\prime}\right)$ of $w^{\prime}$, there exists a neighbourhood $U(s, w)$ of $(s, w)$ such that $F(t, x) \cap V\left(w^{\prime}\right) \neq \varnothing$, for all $(t, x) \in U(s, w)$. The existence proofs for solutions of the differential inclusion (1.1) generally rely on either approximation schemes or a fixed point reformulation.

Differential equations, and their extensions into differential inclusions, are not the only kind of natural dynamics. Some extensions to stochastic cases where also studied, after the pioneering works of Aubin-da Prato [2, 3, 4, 5, and Kisielewicz [18, 19, 20. The works [2, 3, 4, 5] were essentially motivated by studying viability questions in a stochastic setting. M. Kisielewicz in [18] defined the notion of solution and obtained the existence of stochastic differential inclusions of the form

$$
X_{t}-X_{s} \in \int_{s}^{t} F\left(r, X_{r}\right) d r+\int_{s}^{t} G\left(r, X_{r}\right) d W_{r}
$$

where $W$ is an $\mathbb{R}^{\ell}$-valued Brownian motion, $F$ is a lower semicontinuous set-valued random map with values in $\mathbb{R}^{d}$ and $G$ is a lower semicontinuous set-valued map with values in $L\left(\mathbb{R}^{d}, \mathbb{R}^{\ell}\right)$. In [19], M. Kisielewicz also studied the case where equation $(1.2)$ also contains an additional compound Poisson random measure term; the case of semimartingale drivers was also investigated. All proofs fundamentally rely on the isometry property of stochastic integration with respect to Brownian motion or compound Poisson measures. There are however a number of interesting non-semimartingale random processes of practical relevance, such as Mandelbrot's fractional Brownian motion [25] or random Fourier series [14]. Their sample paths are $\alpha$-Hölder continuous, for a regularity exponent $\alpha \in(0,1)$. One can use T. Lyons' work [23] to solve differential equations driven by a fractional Brownian motion, for $\alpha>1 / 2$; it relies on the notion of Young integral [26]. The case $1 / 4 \leqslant \alpha \leqslant 1 / 2$ is much more involved and can be handled using Lyons' theory of rough paths [24]. In [22], A. Levakov and M. Vas'kovskii obtain the existence of solutions to stochastic differential inclusions of the form

$$
X_{t}-X_{s} \in \int_{s}^{t} F\left(r, X_{r}\right) d r+\int_{s}^{t} G_{1}\left(r, X_{r}\right) d W_{r}+\int_{s}^{t} G_{2}\left(r, X_{r}\right) d W_{r}^{\mathrm{FBM}}
$$

where $W^{\mathrm{FBM}}$ is a fractional Brownian motion with $\alpha$-Hölder continuous sample paths, with $\alpha>1 / 2$, and the set valued map $G_{2}$ is a globally Lipschitz function of $x$ that takes values in the set of nonempty compact convex subsets of $\mathbb{R}^{d}$ and satisfies a local $\delta$-Hölder condition as a function of time, with $\delta>1-\alpha$.

\section{2 - Young differential inclusions}

Motivated by control problems, the first aim of this article is to define and prove the existence of solutions of Young differential inclusions

$$
z_{t}=\xi+\int_{0}^{t} v_{r} d x_{r}, \quad v_{r} \in F\left(z_{r}\right)
$$


where $x$ is an $\mathbb{R}^{\ell}$-valued $\alpha$-Hölder continuous control, with $\alpha>1 / 2$, and $F$ is a $\gamma$-Hölder continuous set valued map with compact values, for a regularity exponent $\gamma \in\left(\frac{1}{\alpha}-1,1\right)$. We do not require that $F$ takes values in convex sets. The notion of a solution to a Young differential inclusion involves a number of elementary results on Young integrals that are recalled in Appendix A

Notations. We gather here a number of notations that are used throughout the work.

- For any integer $m$, a point $a \in \mathbb{R}^{m}$ and a positive real number $R$, we denote by $B(a, R)$ the closed ball of center $a$ and radius $R$ in $\mathbb{R}^{m}$.

- For $\alpha \in(0,1]$ and $U, V$ Banach spaces, we denote by $C^{\alpha}(U, V)$ the space of $\alpha$-Hölder continuous functions from $U$ to $V$. We write $\|x\|_{\alpha}$ for the $\alpha$-Hölder semi-norm of a path $x \in C^{\alpha}(U, V)$. We set $x_{s, t}:=x_{t}-x_{s}$, for $s, t \in U$.

- Given a set $S$, we denote by $2^{S}$ the power set of $S, \dot{2}^{S}$ for $2^{S} \backslash\{\varnothing\}$ and $\mathcal{K}(S)$ the compact sets of $\dot{2}^{S}$. We denote by $L\left(\mathbb{R}^{\ell}, \mathbb{R}^{d}\right)$ the space of linear maps from $\mathbb{R}^{\ell}$ to $\mathbb{R}^{d}$. Recall that endowing the space $2^{L\left(\mathbb{R}^{\ell}, \mathbb{R}^{d}\right)}$ with the Hausdorff pseudo-metric turns the space $\mathcal{K}\left(L\left(\mathbb{R}^{\ell}, \mathbb{R}^{d}\right)\right)$ into a complete metric space. For $F: \mathbb{R}^{d} \rightarrow \mathcal{K}\left(L\left(\mathbb{R}^{\ell}, \mathbb{R}^{d}\right)\right)$ a $\gamma$-Hölder set-valued map, we denote again by $\|F\|_{\gamma}$ the $\gamma$-Hölder semi-norm of $F$. (See Chapter 1, Section 5 in [1])

- If $f$ is a single-valued map of $p$-variation, we denote by $\|f\|_{p-v a r}$ the $p$-variation semi-norm of $f$ - more on this notation in Appendix A

Definition - Let $x$ be an element of $C^{\alpha}\left([0, T], \mathbb{R}^{\ell}\right)$, with $\alpha \in\left(\frac{1}{2}, 1\right]$, and $F: \mathbb{R}^{d} \mapsto \dot{2}^{L\left(\mathbb{R}^{\ell}, \mathbb{R}^{d}\right)}$ be a set-valued map. A solution to the Young differential inclusion

$$
d z_{t} \in F\left(z_{t}\right) d x_{t}, \quad z_{0}=\xi \in \mathbb{R}^{d}
$$

is a pair of paths $(z, v)$, defined on the time interval $[0, T]$,

- with $v$ a $L\left(\mathbb{R}^{\ell}, \mathbb{R}^{d}\right)$-valued path of finite $p$-variation such that $\alpha+\frac{1}{p}>1$,

- and for all $0 \leqslant t \leqslant T$,

$$
v_{t} \in F\left(z_{t}\right) \quad \text { and } \quad z_{t}=\xi+\int_{0}^{t} v_{s} d x_{s} .
$$

The letter $v$ is chosen for "velocity". The integral $\int_{0}^{t} v_{s} d x_{s}$ makes sense as a Young integral under the assumption $\alpha+\frac{1}{q}>1-$ see Appendix A

1.1. Theorem - Let a positive time horizon $T$ be given and $x \in C^{\alpha}\left([0, T], \mathbb{R}^{\ell}\right)$ with $\alpha \in\left(\frac{1}{2}, 1\right]$. Let $F: \mathbb{R}^{d} \mapsto \mathcal{K}\left(L\left(\mathbb{R}^{\ell}, \mathbb{R}^{d}\right)\right)$ be a bounded set-valued map with nonempty compact values and $\gamma$-Hölder, for a regularity exponent $\gamma \in\left(\frac{1}{\alpha}-1,1\right)$. Then, for any initial condition $\xi \in \mathbb{R}^{d}$, the Young differential inclusion

$$
d z_{t} \in F\left(z_{t}\right) d x_{t},
$$

has a solution path started from $\xi$, defined over the time interval [0,T].

For a single-valued map, this is a consequence of Young's original result [26, 23]. Unlike the setting of ordinary differential equations, no uniqueness is to be expected in the present setting. Our regularity condition on the set-valued map $F$ is the same as in the ordinary differential setting. However, there may be no Hölder, or even continuous, selection of $F-$ see Proposition 8.2 in [9, so existence results for Young differential inclusions do not follow from existence results for Young differential equations. We refer the reader to Chapters 1 
and 2 of $[1]$ for the basics on differential inclusions. Note that since continuous paths with finite $p$-variation can be reparamatrized into $1 / p$-Hölder paths, the result of Theorem 1.1 holds for continuous paths $x$ with finite $p$-variation $1 \leqslant p<2$.

\section{3 - A selection result}

As a by-product of our proof of Theorem 1.1, we show in Section 3 that a bounded, nonempty compact-valued, $\gamma$-Hölder continuous set-valued map on the interval $[0,1]$ has a selection with finite $p$-variation, for $p>1 / \gamma$.

1.2. Theorem - Pick $\gamma \in(0,1]$, and let $F:[0,1] \mapsto \mathcal{K}\left(\mathbb{R}^{d}\right)$, be a bounded, $\gamma$-Hölder nonempty compact set-valued map. Then, for any $p>1 / \gamma$ and any $\xi \in F(0)$, there exists a map $f:[0,1] \mapsto \mathbb{R}^{d}$, of finite $p$-variation, such that $f(t) \in F(t)$, for all $0 \leqslant t \leqslant 1$, with $f(0)=\xi$, and which furthermore satisfies the estimate for a constant $C_{\gamma, p}$ depending only on $\gamma, p$

$$
\|f\|_{p-\operatorname{var}} \leqslant C_{\gamma, p}\|F\|_{\gamma}
$$

This statement partly answers an open question in Chistyakov and Galkin's work [9]see Remark 8.1 therein.

\section{4 - Rough differential inclusions}

Stochastic analysis has undergone a real change under the impulse of T. Lyons' theory of rough paths [24]. It provides a deep understanding of stochastic differential equations and disentangles completely in that setting probabilistic and dynamical matters. As a first step to establishing a full theory of rough differential inclusions, the second aim of the present work is to deal with rough differential equations perturbed by set-valued drifts. Fix a finite positive time horizon $T$. We refer here the reader to Appendix A for basics on rough paths and rough integrals, in the setting of controlled paths; it suffices here to say that controlled paths are needed to make sense of the rough integral that appears in the next definition.

For an integer $k \geqslant 1$ and $\gamma \in[0,1]$, let us denote by $C_{b}^{k, \gamma}$ the space of bounded maps $G$ from $\mathbb{R}^{d}$ to $L\left(\mathbb{R}^{\ell}, \mathbb{R}^{d}\right)$ which are $k$-times differentiable, with bounded derivatives, and have a $\gamma$-Hölder $k$-th derivative. We endow $C_{b}^{k, \gamma}$ with the norm

$$
\|G\|_{C_{b}^{k, \gamma}}:=\sum_{i=0}^{k}\left\|D^{i} G\right\|_{\infty}+\|G\|_{\gamma} \mathbf{1}_{\gamma>0},
$$

where $D^{i} G$ is the $i$-th derivative of $G$. By convention $D^{0} G:=G$.

Definition - Let $T$ be a fixed positive time horizon. Pick $\xi \in \mathbb{R}^{d}$, let $\Omega$ be a subset of $\mathbb{R} \times \mathbb{R}^{d}$ containing $[0, T] \times\{\xi\}$. Let $\mathbf{X}$ be a weak geometric $\alpha$-Hölder rough path, with $1 / 3<\alpha \leqslant 1 / 2$. Let $F: \Omega \rightarrow \dot{2}^{\mathbb{R}^{d}}$, be a set-valued map, and $G \in C_{b}^{1, \gamma}$ with $(2+\gamma) \alpha>1$. An $\mathbb{R}^{d}$-valued path $z$ started from $\xi$ is said to be a solution to the rough differential inclusion

$$
d z_{t} \in F\left(t, z_{t}\right) d t+G\left(z_{t}\right) d \mathbf{X}_{t},
$$

if $z$ is part of a path $\left(z, z^{\prime}\right)$ controlled by $\mathbf{X}$, and there exists an absolutely continuous path $x$ from $[0, T]$ to $\mathbb{R}^{d}$ starting from zero, such that

$$
\dot{x}_{t} \in F\left(t, z_{t}\right)
$$


at Lebesgue almost all times $0 \leqslant t \leqslant T$, and one has

$$
z_{t}=\xi+x_{t}+\int_{0}^{t} G\left(z_{s}\right) d \mathbf{X}_{s}
$$

at all times.

The regularity assumption on $G$ is the optimal classical regularity assumption required to have a well-posed rough differential equation when $F$ is null. The technics used to prove the existence of a solution to equation (1.5) are different depending on the regularity of the set-valued drift $F$. We first deal with the case where the drift is upper semicontinuous. We need in that case to assume that $F$ takes values in the set of convex compact subsets of $\mathbb{R}^{d}$.

1.3. Theorem - Let $\mathbf{X}$ be a weak geometric $\alpha$-Hölder rough path, with $1 / 3<\alpha \leqslant 1 / 2$, and let $G$ be $a C_{b}^{2, \gamma}$ one form, with $\alpha(2+\gamma)>1$. Let $F$ be an upper semicontinuous set-valued drift. Assume further that $F$ is locally bounded, and takes it values in the set of nonempty compact convex subsets of $\mathbb{R}^{d}$. Then there exists a time horizon $T^{*} \in(0, T)$ and a solution $z$ to the rough differential inclusion (1.5), defined on the time interval $\left[0, T^{*}\right]$.

In contrast to the preceding statement, we do not need to assume that $F$ has convex images in the case where it is lower semicontinuous; we assume instead a mild boundedness assumption on $F$.

1.4. Theorem - Let $\mathbf{X}$ be a weak geometric $\alpha$-Hölder rough path, with $1 / 3<\alpha \leqslant 1 / 2$, and let $G$ be $a C_{b}^{1, \gamma}$ one form, with $\alpha(2+\gamma)>1$. Assume that the set-valued drift $F$ is defined on a closed subset $\Omega$ of $\mathbb{R} \times \mathbb{R}^{d}$, where it is lower semicontinuous. Assume further that $F$ takes values in the set $\mathcal{K}\left(\mathbb{R}^{d}\right)$ of nonempty compact subsets of $\mathbb{R}^{d}$, and that there is a positive constant $L$ such that $[0, T] \times B\left(\xi, L T^{\alpha}\right) \subset \Omega$, and

$$
\|F(t, a)\| \leqslant L, \quad \forall(t, a) \in[0, T] \times B\left(\xi, L T^{\alpha}\right) .
$$

Then there exists a time horizon $T^{*} \in(0, T)$ and a solution $z$ to the rough differential inclusion (1.5) stated at $\xi$, defined on the time interval $\left[0, T^{*}\right]$.

Note that the regularity of $G$ is lower in Theorem 1.4 than in Theorem 1.3 . When $G=0$ in Theorem 1.3 and Theorem 1.4, we recover classical conditions for existence of solutions to differential inclusions - see e.g. the classical reference [1]. In Theorem 1.4, when $F=\{0\}$, we recover the optimal condition of regularity on $G$ for existence of solutions to a rough differential equation [12].

The work has been organized as follows. Section 2 is dedicated to proving Theorem 1.1 on Young differential inclusions, following the above strategy. Theorem 1.2 is proved in Section 3 , and Section 4 is dedicated to the proof of Theorem 1.4 .

\section{2 - Young differential inclusions}

This section is dedicated to proving Theorem 1.1. Given $q \geqslant 1$ and a finite dimensional vector space $E$, we denote by $V_{q}([0, T], E)$ the space of $E$-valued paths with finite $q$ variation on the time interval $[0, T]$. Refer to Appendix A

We fix $\alpha \in\left(\frac{1}{2}, 1\right]$ once and for all in this section, and work in the setting of Theorem 1.1. The pattern of proof of Theorem 1.1 goes as follows. Recall from Appendix $\mathrm{A}$ that the space of paths with finite $p$-variation is endowed with the $p$-variation norm $\|\cdot\|_{p \text {-var, } \infty}$, defined in A.1. Given a positive finite time horizon $T$, we define the dyadic partitions 
$\pi^{(m)}=\left\{t_{i}^{m}\right\}$ of the interval $[0, T]$, with $t_{i}^{m}:=i 2^{-m} T, 0 \leqslant i \leqslant 2^{m}$ and $m \geqslant 1$. Let $\gamma$ be given in Theorem 1.1 and $p \geqslant 1$ be such that $\frac{\gamma}{p}+\alpha>1$. We construct in Section 2.1 an approximate solution to the problem on a sufficiently small time interval $[0, T]$, under the form of a pair $\left(z^{m}, v^{m}\right)$ such that

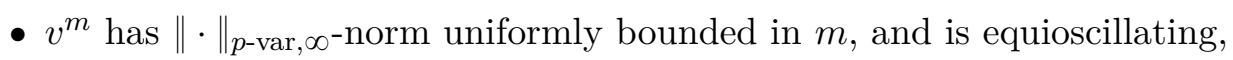

- $v_{t}^{m} \in F\left(z_{t}^{m}\right)$ for all dyadic times, and

$$
z_{t}^{m}=\xi+\int_{0}^{t} v_{u}^{m} d x_{u}, \quad 0 \leqslant t \leqslant T,
$$

with $\xi \in \mathbb{R}^{d}$.

It follows then from the first item that the sequence $v^{m}$ has a converging subsequence

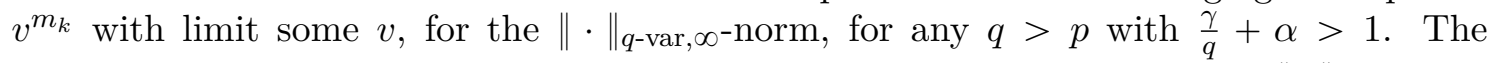
continuity statement from Corollary A.2 implies then that $z^{m_{k}}$ converges in $\|\cdot\|_{\alpha, \infty}$-norm to the path $z:=\xi+\int_{0}^{\cdot} v_{u} d x_{u}$. One gets the fact that $v_{t} \in F\left(z_{t}\right)$, for all $0 \leqslant t \leqslant T$, from the fact that $F$ is bounded and takes values in closed sets. Thus $(z, v)$ is a solution in $C^{\alpha}\left([0, T], \mathbb{R}^{d}\right) \times V_{q}\left([0, T], L\left(\mathbb{R}^{\ell}, \mathbb{R}^{d}\right)\right)$. The existence of a solution to the inclusion defined up to the initial time horizon is a consequence of the fact that the previous existence time does not depend on $\xi$. We now turn to the details.

\section{1 - Construction of the approximate solution}

For $t \in \bigcup_{m \geqslant 0} \pi^{(m)}$, set

$$
M(t):=\min \left\{j ; t \in \pi^{(j)}\right\}
$$

and define the ancestor $s(t)$ of $t$ as

$$
s(t):=\max \left\{s \in \pi^{(M(t)-1)} ; s<t\right\} .
$$

For each $m \geqslant 0$, we construct the path $z^{m}$ on $\left[0, t_{i+1}^{m}\right]$, and $v^{m}$ on $\left[0, t_{i+1}^{m}\right)$, recursively on $0 \leqslant i \leqslant 2^{m}-1$ where $t_{i+1}^{m}=2^{-m} T(i+1)$. The construction is not inductive on $m$.

- For $m=0$, choose $v_{0}^{0} \in F(\xi)$, and set

$$
v_{t}^{0}=v_{0}^{0}, \quad z_{t}^{0}=\xi+v_{0}^{0} x_{0, t}, \quad \forall t \in[0, T] .
$$

- Pick $m \geqslant 1$.We set

$$
\begin{aligned}
& v_{t}^{m}:=v_{0}^{0}, \quad \forall t \in\left[0, T 2^{-m}\right), \\
& z_{t}^{m}:=\xi+v_{0}^{0} x_{0, t}, \quad \forall t \in\left[0, T 2^{-m}\right] .
\end{aligned}
$$

This starts the induction over $0 \leqslant i \leqslant 2^{m}-1$. If $z^{m}:\left[0, t_{i}^{m}\right] \rightarrow \mathbb{R}^{d}$ and $v^{m}:\left[0, t_{i}^{m}\right) \rightarrow \mathbb{R}^{d}$, have been constructed, use the Hölder continuity of $F$ to choose $v_{t_{i}^{m}}^{m} \in F\left(z_{t_{i}^{m}}^{m}\right)$ such that

$$
\left|v_{t_{i}^{m}}^{m}-v_{s\left(t_{i}^{m}\right)}^{m}\right| \leqslant\|F\|_{\gamma}\left|z_{t_{i}^{m}}^{m}-z_{s\left(t_{i}^{m}\right)}^{m}\right|^{\gamma},
$$

and set

$$
\begin{aligned}
& v_{t}^{m}:=v_{t_{i}^{m}}^{m}, \quad \forall t \in\left[t_{i}^{m}, t_{i}^{m}+T 2^{-m}\right), \\
& z_{t}^{m}:=z_{t_{i}^{m}}^{m}+v_{t_{i}^{m}}^{m} x_{t_{i}^{m}}, \quad \forall t \in\left[t_{i}^{m}, t_{i}^{m}+T 2^{-m}\right] .
\end{aligned}
$$

If $t_{i}^{m}+2^{-m} T=T$, set $v_{T}^{m}=v_{t_{i}^{m}}^{m}$. 
We have $z^{m}=\xi+\int_{0}^{t} v_{u}^{m} d x_{u}$, as a consequence of the fact that $v^{m}$ is constant along the intervals of the partition $\pi^{(m)}$ of $[0, T]$. The next section is dedicated to proving a uniform $p$-variation bound on a small time interval satisfied uniformly by the paths $v^{m}$.

\section{2 - Study of the $p$-variation norm of $v^{m}$ in a small time interval}

This section is dedicated to proving the following intermediate result.

2.1. Proposition - Pick $\frac{1}{1+\gamma}<\beta<\alpha$, and set $T_{0}$ as equal to

$$
\min \left\{1,\left(2\|F\|_{\infty}\|x\|_{\alpha}\right)^{-\frac{2}{\alpha-\beta}},\left(\frac{2\|F\|_{\gamma}\|x\|_{\alpha}}{1-2^{-(\alpha+\beta \gamma-1)}}\right)^{-\frac{2}{\alpha-\beta}},\left(\frac{\|F\|_{\gamma}}{1-2^{-\gamma \beta}}\right)^{\frac{-4}{\gamma(\alpha-\beta)}}\right\} .
$$

Pick $p>1 /(\gamma \beta)$. Then we have, for any $S \leqslant T_{0}$,

$$
\left\|v^{m}\right\|_{p-\operatorname{var},[0, S]} \leqslant\left(\frac{1}{1-2^{-\gamma \beta p}}\right)^{1 / p} S^{\frac{(\alpha-\beta) \gamma}{4}+\gamma \beta} .
$$

The proof of Proposition 2.1 proceeds in several steps. We first give a discrete integral representation of $z^{m}$ that proves useful.

2.2. Lemma - Pick $0 \leqslant n \leqslant m$, and two consecutive points $s, t$ in $\pi^{(n)}$. Then, setting $s_{i}^{k}=s+i 2^{-n-k} T$, we have

$$
z_{s, t}^{m}=v_{s}^{m} x_{s, t}+\sum_{k=0}^{m-n-1} \sum_{i=0}^{2^{k}-1} v_{s_{i}^{k}, s_{2 i+1}^{k+1}}^{m} x_{s_{2 i+1}^{k+1}, s_{i+1}^{k}} .
$$

Proof. For $0 \leqslant s \leqslant t \leqslant T$, set

$$
\mu_{s, t}^{m}:=\mu_{s, t}^{m,(0)}=v_{s}^{m} x_{s, t}
$$

and, for $k \geqslant 1$,

$$
\mu_{s, t}^{m,(k)}:=\mu_{s, \frac{t+s}{2}}^{m,(k-1)}+\mu_{\frac{t+s}{2}, t}^{m,(k-1)} .
$$

- We first prove by descending induction on $n$ that for $0 \leqslant n \leqslant m$, and two consecutive points $s, t$ in $\pi^{(n)}$, we have

$$
z_{s, t}^{m}=\mu_{s, t}^{m,(m-n)}=\mu_{s, t}^{m,(k)}, \quad \forall k \geqslant m-n .
$$

This identity holds true when $n=m$ as a consequence of the definition of the objects. Assume that (2.4) holds true for $n \leqslant m$, and let $s, t$ be two consecutive points in $\pi^{(n-1)}$. Since $\left(s, \frac{s+t}{2}\right)$ and $\left(\frac{s+t}{2}, t\right)$ are two consecutive pairs of points in $\pi^{(n)}$, we have, from the definition of $\mu^{m,(k)}$ and induction,

$$
z_{s, t}^{m}=z_{s, \frac{s+t}{2}}^{m}+z_{\frac{s+t}{2}, t}^{m}=\mu_{s, t}^{m,(m-n+1)} .
$$

For $k \geqslant(m-n)$, we have

$$
\mu_{s, t}^{m,(k)}=\mu_{s, \frac{s+t}{2}}^{m,(k-1)}+\mu_{\frac{s+t}{2}, t}^{m,(k-1)}=\mu_{s, \frac{s+t}{2}}^{m,(m-n)}+\mu_{\frac{s+t}{2}, t}^{m,(m-n)}=\mu_{s, t}^{m,(m-n+1)} ;
$$

this closes the inductive proof of identity 2.4

- One then sees by induction on $k$ that setting as in the statement of the Lemma

$$
s_{i}^{k}=s+i 2^{-k}(t-s)=s+i 2^{-n-k} T,
$$


one has

$$
\mu_{s, t}^{m,(k)}=\sum_{i=0}^{2^{k}-1} \mu_{s_{i}^{k}, s_{i+1}^{k}}^{m}
$$

and

$$
\mu_{s, t}^{m,(k+1)}-\mu_{s, t}^{m,(k)}=\sum_{i=0}^{2^{k}-1} v_{s_{i}^{k}, s_{2 i+1}^{k+1}}^{m} x_{s_{2 i+1}^{k+1}, s_{i+1}^{k}} .
$$

Summing equation (2.5) for $k$ from 0 to $m-n$, and using identity (2.4) gives the identity of the Lemma.

2.3. Corollary - Pick $\frac{1}{1+\gamma}<\beta<\alpha$, and set

$$
T_{1}:=\min \left\{1,\left(2\|F\|_{\infty}\|x\|_{\alpha}\right)^{-\frac{2}{\alpha-\beta}},\left(\frac{2\|F\|_{\gamma}\|x\|_{\alpha}}{1-2^{-(\alpha+\beta \gamma-1)}}\right)^{-\frac{2}{\alpha-\beta}}\right\} .
$$

Then we have for any $0 \leqslant S \leqslant T_{1}$ and any $0 \leqslant n \leqslant m$, the $m$-uniform bound

$$
\sup _{[s, t] \in \pi^{(n)}}\left|z_{s, t}^{m}\right| \leqslant S^{\frac{\alpha-\beta}{2}}\left(S 2^{-n}\right)^{\beta}
$$

the supremum is over consecutive points $s, t$ of $\pi^{(n)}$.

Proof. The proof is again by descending induction on $n \in\{0, \ldots, m\}$. We first have for two consecutive points $s, t$ of $\pi^{(m)}$ the estimate

$$
\left|z_{s, t}^{m}\right| \leqslant\|F\|_{\infty}\|x\|_{\alpha}\left(S 2^{-m}\right)^{\alpha}
$$

so (2.6) holds true for $m=n$ since $0 \leqslant S \leqslant T_{1}$. Assume now that 2.6 has been proved for $n \leqslant m$, an let $s, t$ be two consecutive points of $\pi^{(n-1)}$. We use the representation formula

$$
z_{s, t}^{m}=v_{s}^{m} x_{s, t}+\sum_{k=0}^{m-n+1} \sum_{i=0}^{2^{k}-1} v_{s_{i}^{k}, s_{2 i+1}^{k+1}}^{m} x_{s_{2 i+1}^{k+1}, s_{i+1}^{k}}
$$

from Lemma 2.2 , with $s_{i}^{k}=s+i 2^{-n-k+1} S$. Note that $M\left(s_{2 i+1}^{k+1}\right)=n+k$, here, and the ancestor $s\left(s_{2 i+1}^{k+1}\right)$ of $s_{2 i+1}^{k+1}$ is $s_{i}^{k}$. Then, using (2.6) for $n+k$, we have

$$
\left|v_{s_{i}^{k}, s_{2 i+1}^{k+1}}^{m}\right| \leqslant\|F\|_{\gamma}\left|z_{s_{i}^{k}, s_{2 i+1}^{k+1}}^{m}\right|^{\gamma} \leqslant\|F\|_{\gamma} S^{\frac{(\alpha-\beta)}{2}} \gamma\left(S 2^{-n-k}\right)^{\beta \gamma},
$$

so we obtain for $\left|z_{s t}^{m}\right|$ the upper bounds

$$
\begin{aligned}
& \|F\|_{\infty}\|x\|_{\alpha}\left(2^{-n+1} S\right)^{\alpha}+\sum_{k=0}^{m-n+1} 2^{k} S^{\frac{(\alpha-\beta)}{2}} \gamma\left(S 2^{-n-k}\right)^{\beta \gamma}\|x\|_{\alpha}\left(S 2^{-n-k}\right)^{\alpha} \\
& \leqslant\|F\|_{\infty}\|x\|_{\alpha}\left(2^{-n+1} S\right)^{\alpha}+\|x\|_{\alpha}\|F\|_{\gamma} S^{\alpha+\gamma \beta+\frac{(\alpha-\beta)}{2} \gamma} \frac{2^{-n(\alpha+\beta \gamma)}}{1-2^{-(\alpha+\beta \gamma-1)}} .
\end{aligned}
$$

The choice of $S \leqslant T_{1}$ ensures that

$$
\|F\|_{\infty}\|x\|_{\alpha}\left(2^{-n+1} S\right)^{\alpha} \leqslant \frac{1}{2} S^{\frac{\alpha-\beta}{2}}\left(2^{-(n-1)} S\right)^{\beta}
$$

and since $\alpha+\gamma \beta>1>\alpha$ and $S<1$

$$
\|x\|_{\alpha}\|F\|_{\gamma} S^{\alpha+\gamma \beta+\frac{(\alpha-\beta)}{2} \gamma} \frac{2^{-n(\alpha+\beta \gamma)}}{1-2^{-(\alpha+\beta \gamma-1)}} \leqslant \frac{1}{2} S^{\frac{\alpha-\beta}{2}}\left(2^{-(n-1)} S\right)^{\beta}
$$


this closes the descending induction step.

Recall the oscillation $\operatorname{Osc}(v, I)$ of a function $v: I \mapsto \mathbb{R}^{d}$, is defined by the formula

$$
\operatorname{Osc}(v, I):=\sup _{a, b \in I}(v(b)-v(a)) .
$$

The uniform control of the oscillation of the $v^{m}$ provided by the next statement is necessary to use the compactness result on the set of bounded functions equipped with uniform norm stated in Theorem 5, Section 4, Chapter 0 of Aubin and Cellina's book [1. Recall the definition of $T_{0} \leqslant T_{1}$ from the statement of Proposition 2.1. The notation $[s, t] \in \pi^{(n)}$ used below stands for two consecutive points $s, t$ in $\pi^{(n)}$.

2.4. Corollary - For any $0 \leqslant S \leqslant T_{0}$, we have, for any $0 \leqslant n \leqslant m$,

$$
\sup _{[s, t] \in \pi^{(n)}} \operatorname{Osc}\left(v^{m},[s, t)\right) \leqslant S^{\frac{(\alpha-\beta) \gamma}{4}}\left(S 2^{-n}\right)^{\gamma \beta} .
$$

Proof. Note that if $n \geqslant m$ and $s$ and $t$ are two consecutive points of $\pi^{(n)}$, the function $v^{m}$ has null oscillation on the interval $[s, t)$, since it is constant on the intervals of the partition $\pi^{(m)}$. Let then take $n \leqslant(m-1)$. Set $s_{0}=s$ and define a finite sequence $\left(s_{i}\right)_{i=0, \ldots, m-n}$ setting $s_{i+1}=s_{i}+2^{-(n+i+1)} S$, if $s_{i}+2^{-(n+i+1)} S \leqslant t$, and $s_{i+1}=s_{i}$, otherwise. Then $s_{i} \in \pi^{(n+i)}$, for any $i$, and either $s_{i+1}=s_{i}$ or its ancestor $s\left(s_{i+1}\right)$ is $s_{i}$. We then have from the uniform estimate 2.6 on $z_{s, t}^{m}$ the bound

$$
\left|v_{s_{i}, s_{i+1}}^{m}\right| \leqslant\|F\|_{\gamma}\left|z_{s_{i}, s_{i+1}}^{m}\right|^{\gamma} \leqslant\|F\|_{\gamma}\left(S^{\frac{\alpha-\beta}{2}}\left(S 2^{-n-i}\right)^{\beta}\right)^{\gamma} .
$$

We obtain (2.7) summing these inequalities for $i$ from 0 to $(m-n)$, and from the definition of $T_{0}$.

Proof of Proposition 2.1. Take $0 \leqslant S \leqslant T_{0}$, and let $\pi=\left(s_{i}\right)_{i=0}^{N}$ be a partition of the interval $[0, S]$.

The following partition is a kind of greedy partition of the set $\{0, \ldots, N-1\}$, in terms of the size of corresponding increments in the above formula. Let then set

$$
\begin{aligned}
& I_{0}:=\left\{\ell \in\{0, . ., N-1\} ; \exists j \in\left\{0, \ldots, 2^{m}-1\right\},\left(s_{\ell}, s_{\ell+1}\right] \subset\left[t_{j}^{m}, t_{j+1}^{m}\right)\right\}, \\
& I_{1}:=\left\{\ell \in\{0, . ., N-1\} ; 2^{-1} \in\left(s_{\ell}, s_{\ell+1}\right]\right\},
\end{aligned}
$$

and, for $2 \leqslant j \leqslant m$, set

$$
I_{j}:=\left\{\ell \in\{0, . ., N-1\} ; \exists t \in \pi^{(j)} \backslash \pi^{(j-1)} \cap\left(s_{\ell}, s_{\ell+1}\right]\right\} .
$$

We define a partition of $\{0, \ldots, N-1\}$ setting $K_{0}:=I_{0}$ and

$$
K_{j}:=I_{j} \backslash \bigcup_{k=0}^{j-1} I_{k},
$$

for $2 \leqslant j \leqslant m$. Note that $K_{j}$ has at most $2^{j}$ elements.

- For $\ell \in K_{1}$, we know from Corollary 2.4 on oscillations of $v^{m}$, with $n=0$, that

$$
\left|v_{s_{l+1}}^{m}-v_{s_{l}}^{m}\right| \leqslant S^{\frac{(\alpha-\beta) \gamma}{4}+\gamma \beta} .
$$

- For $\ell \in K_{0}$, one has $v_{s_{j}, s_{j+1}}^{m}=0$, since $v^{m}$ is constant over the intervall of $\pi^{(m)}$. 
- For $\ell \in K_{j}$, there exists $t \in \pi^{(j)} \backslash \pi^{(j-1)}$ such that $s_{\ell}<t \leqslant s_{\ell+1}$, and for all $u \in \pi^{(j-1)}$, one has either $u \leqslant s_{\ell}$, or $u>s_{\ell+1}$.

Then $\left[s_{\ell}, s_{\ell+1}\right) \subset\left[t-S 2^{-j}, t+S 2^{-j}\right)$, and using Corollary 2.4 with $j-1$, one gets

$$
\left|v_{s_{\ell+1}}^{m}-v_{s_{\ell}}^{m}\right| \leqslant S^{\frac{(\alpha-\beta) \gamma}{4}}\left(S 2^{-(j-1)}\right)^{\gamma \beta} .
$$

Taking $p>1 /(\gamma \beta)$, one then has

$$
\sum_{i=0}^{N-1}\left|v_{s_{i+1}}^{m}-v_{s_{i}}^{m}\right|^{p} \leqslant 2 S^{\frac{(\alpha-\beta) \gamma p}{4}} \sum_{j=0}^{m} 2^{j}\left(S 2^{-(j-1)}\right)^{\gamma \beta p} \leqslant 2 S^{\frac{(\alpha-\beta) \gamma p}{4}} S^{\gamma \beta p} \frac{1}{1-2^{-\gamma \beta p}},
$$

from which 2.3 follows

$$
\left\|v^{m}\right\|_{p-\operatorname{var},[0, S]} \leqslant\left(\frac{1}{1-2^{-\gamma \beta p}}\right)^{1 / p} S^{\frac{(\alpha-\beta) \gamma}{4}+\gamma \beta} .
$$

\section{3 - Local and global existence for solutions}

We finally give the proof of Theorem 1.1 in this section. We first prove the existence of a solution to the differential inclusion (1.4) on the time interval $\left[0, T_{0}\right]$, for the time $T_{0}$ defined in 2.2 in Proposition 2.1. Since the definition of $T_{0}$ does not involve the initial condition $\xi$ of the dynamics, we obtain by concatenation a solution to the inclusion defined over the whole interval $[0, T]$.

- Recall that a sequence of bounded functions $\left(y^{m}\right)_{m \geqslant 0}$ from compact segment $[a, b]$ into a compact set $K$ is said to be equioscillating if, and only if, one can associate to any positive $\varepsilon$ a finite partition $\left(J_{k}\right)_{0 \leqslant k \leqslant r}$ of $[a, b]$ into subintervals such that $\operatorname{Osc}\left(y^{m}, J_{k}\right) \leqslant \varepsilon$, uniformly in $k, m$. The Ascoli-Arzela-type convergence theorem from Theorem 5 of section 4 of chap 0 of Aubin and Cellina's book [1], ensure the existence of a subsequence $\left(y^{m_{k}}\right)_{k \geqslant 0}$ that converges uniformly to some limit bounded function $y$. Since the family $\left(v^{m}\right)_{m \geqslant 0}$ is bounded by $\|F\|_{\infty}$, and equioscillating, from Corolary 2.4, it has a uniformly converging subsequence, with limit $v$.

Pick then $q>p$ such that $\frac{1}{q}+\alpha>1$. Since all the $v^{m}$ have the same starting point, we have the elementary interpolation bound

$$
\begin{aligned}
& \left\|v^{m}-v^{n}\right\|_{q-\operatorname{var},\left[0, T_{0}\right]} \\
& \leqslant\left(2\left\|v^{m}-v^{n}\right\|_{\infty,\left[0, T_{0}\right]}\right)^{\frac{q-p}{q}}\left(\left\|v^{m}\right\|_{p-\operatorname{var},\left[0, T_{0}\right]}+\left\|v^{n}\right\|_{p-\operatorname{var},\left[0, T_{0}\right]}\right)^{p / q},
\end{aligned}
$$

on which we read off the convergence of a subsequence $z^{m_{k}}$ of the $v^{m}$ in $q$-variation norm to its limit, as a consequence of the uniform bound from Proposition 2.1. The convergence to $v$ of this subsequence is thus in the sense of the $\|\cdot\|_{q-\operatorname{var},\left[0, T_{0}\right], \infty}$-norm.

- The continuity result on Young integrals recalled in Corollary A.2 from Appendix A implies then the convregence in the norm $\|\cdot\|_{\alpha, \infty}$ of $z^{m_{k}}$ on $\left[0, T_{0}\right]$ to the path $z$ defined by the equation

$$
z_{t}=\xi+\int_{0}^{t} v_{u} d x_{u}
$$

- It remains to prove that $v_{t} \in F\left(z_{t}\right)$, for all times $0 \leqslant t \leqslant T_{0}$. For a dyadic time $t \in \bigcup_{m \geqslant 0} \pi^{(m)}$, then for $k$ big enough, one has $v_{t}^{m_{k}} \in F\left(z_{t}^{m_{k}}\right)$. Then, since $F$ is $\gamma$-Hölder, 
one has

$$
d\left(v_{t}, F\left(z_{t}\right)\right) \leqslant\left|v_{t}-v_{t}^{m_{k}}\right|+d\left(F\left(z_{t}^{m_{k}}\right), F\left(z_{t}\right)\right) \leqslant\left|v_{t}-v_{t}^{m_{k}}\right|+\|F\|_{\gamma}\left|z_{t}^{m_{k}}-z_{t}\right|^{\gamma}
$$

so one gets $d\left(v_{t}, F\left(z_{t}\right)\right)=0$, and $v_{t} \in F\left(z_{t}\right)$, since $F\left(z_{t}\right)$ is closed. For an non-dyadic time $t \in\left[0, T_{0}\right]$, there exists two consecutive points in $\pi^{(m)}$ such that $t \in[u, v[$, for every $m \geqslant 0$. Then,

$$
\begin{aligned}
d\left(v_{t}, F\left(z_{t}\right)\right) \leqslant\left|v_{t}-v_{t}^{m}\right| & +\left|v_{t}^{m}-v_{u}^{m}\right|+d\left(v_{u}^{m}, F\left(z_{u}^{m}\right)\right)+d\left(F\left(z_{u}^{m}\right), F\left(z_{u}\right)\right) \\
& +d\left(F\left(z_{u}\right), F\left(z_{t}\right)\right),
\end{aligned}
$$

while we have from Corollary 2.4 and Corollary 2.3

$$
d\left(v_{t}, F\left(z_{t}\right)\right) \leqslant\left|v_{t}-v_{t}^{m}\right|+\left(T_{0} 2^{-m}\right)^{\gamma \beta}+\|F\|_{\gamma}\left|z_{u}^{m}-z_{u}\right|^{\gamma}+\|F\|_{\gamma}\left|z_{u}-z_{t}\right|^{\gamma}
$$

and

$$
\begin{aligned}
d\left(v_{t}, F\left(z_{t}\right)\right) \leqslant & \left\|v-v^{m_{k}}\right\|_{\infty,[0, T]}+\left(T_{0} 2^{-m_{k}}\right)^{\gamma \beta} \\
& +\|F\|_{\gamma}\left(\left\|z-v^{m_{k}}\right\|_{\infty,[0, T]}+\|z\|_{\alpha,[0, T]}\left(T_{0} 2^{-m_{k}}\right)^{\gamma \alpha}\right) .
\end{aligned}
$$

So one gets $d\left(v_{t}, F\left(z_{t}\right)\right)=0$, and $v_{t} \in F\left(z_{t}\right)$, since $F\left(z_{t}\right)$ is closed.

\section{3 - A selection result}

As a by product of the proof of Theorem 1.1, we obtain the selection result of Theorem 1.2 wich partially answers Remark 8.1 of Chistyakov and Galkin's work 9]. This section is dedicated to proving Theorem 1.2 .

We use the same notations an in Section 2. Define for each non-negative integer $m$ the partition $\pi^{m}:=\left\{t_{i}^{m}\right\}_{i=0.2^{m}}$ of the interval [0,1], with $t_{i}^{m}:=i 2^{-m}$. We define as follows a path $x^{m}:[0,1] \mapsto \mathbb{R}^{d}$, on each sub-interval $\left[0, t_{i}^{m}\right)$, recursively over $i$.

- For $m=0$, set $x^{0}(t)=x_{0}$, for all $0 \leqslant t \leqslant 1$.

- For $m \geqslant 1$, set first $x^{m}(t)=x_{0}$, on $\left[0, t_{1}^{m}\right)$, and assuming $x^{m}$ has been constructed on the time interval $[0, \tau)$, set $x_{T}^{m}=x_{\tau-2^{-m}}^{m}$, if $\tau=T$, otherwise choose $x_{\tau}^{m} \in F(\tau)$ such that

$$
d\left(x_{\tau}^{m} ; x_{s(\tau)}^{m}\right) \leqslant\|F\|_{\gamma} 2^{(-M(\tau)+1) \gamma}
$$

and set $x_{t}^{m}=x_{\tau}^{m}$, for $\tau \leqslant t<\tau+2^{-m}$.

We first prove that we have for all $r, m$ the estimate

$$
\max _{t \in \pi^{r}} \sup _{s \in\left[t, t+2^{-r}\right)}\left|x_{s, t}^{m}\right| \leqslant \frac{\|F\|_{\gamma}}{1-2^{-\gamma}} 2^{-r \gamma} .
$$

By construction, $x^{m}$ is constant on the each interval of $\pi^{m}$, so if $r \geqslant m, t \in \pi^{r}$ and $s \in\left[t, t+2^{-r}\right)$, then $x_{s, t}^{m}=0$ and (3.1) holds true. Let then consider the case where $r<m$. We define a finite sequence of times $\left(s_{i}\right)_{i=0, \ldots, m-r}$, with $s_{0}=t$, such that for $0 \leqslant i \leqslant(m-r-1)$, we have $s_{i+1}=s_{i}+2^{-r-i-1}$, if $s_{i}+2^{-r-i-1} \leqslant s$, and $s_{i+1}=s_{i}$ otherwise. Then, for $s_{i} \in \pi^{r+i}$, for $i \geqslant 1$, and either $s\left(s_{i+1}\right)=s_{i}$ or $s_{i+1}=s_{i}$. The path $x^{m}$ is constructed in such a way as to have

$$
\left|x_{s_{i}, s_{i+1}}^{m}\right| \leqslant\|F\|_{\gamma} 2^{-(r+i-1) \gamma} .
$$


Summing these estimates for $0 \leqslant i \leqslant m-r$, gives $(3.1)$, and

$$
\max _{t \in \pi^{r}} \operatorname{Osc}\left(x^{m},\left[t, t+2^{-r}\right)\right) \leqslant \frac{2\|F\|_{\gamma}}{1-2^{-\gamma}} 2^{-r \gamma} .
$$

Since $x^{m}$ is constant along the sub-intervals of the partition $\pi^{m}$ it is enough, to compute the $p$-variation of $x^{m}$ along any partition $\pi=\left\{s_{i}, i=0, \ldots, N\right\}$ of $[0,1]$, to assume that all the partition times $s_{i} \in \pi^{(m)}$. Let then define as follows be a finite partition $\left(K_{j}\right)_{j=0, \ldots, m}$ of $\{0, \ldots, N-1\}$, with possibly empty sets :

$$
\begin{aligned}
& I_{0}:=\left\{\ell \in\{0, . ., N-1\} ; \exists j \in\left\{0, \ldots, 2^{m}-1\right\},\left(s_{\ell}, s_{\ell+1}\right] \subset\left[t_{j}^{m}, t_{j+1}^{m}\right)\right\}, \\
& I_{1}:=\left\{\ell \in\{0, \ldots, N-1\} ; 2^{-1} \in\left(s_{\ell}, s_{\ell+1}\right]\right\}, \\
& I_{j}:=\left\{\ell \in\{0, \ldots, N-1\} ; \exists t \in \pi^{j} \backslash \pi^{j-1}, t \in\left(s_{\ell}, s_{\ell+1}\right]\right\}, \quad 2 \leqslant j \leqslant m,
\end{aligned}
$$

with $K_{0}:=I_{0}$ and

$$
K_{j+1}:=I_{j+1} \backslash\left(\bigcup_{k=0}^{j} I_{k}\right) .
$$

For $\ell \in K_{1}$, using $(3.2)$ for $r=0$ we bound

$$
\left|x_{s_{\ell+1}}^{m}-x_{s_{\ell}}^{m}\right| \leqslant \frac{2\|F\|_{\gamma}}{1-2^{-\gamma}} .
$$

For $\ell \in K_{j}$, then there exists $t \in \pi^{j} \backslash \pi^{j-1}$ such that

$$
s_{\ell}<t \leqslant s_{\ell+1},
$$

and any $u \in \pi^{j-1}$ satisfies either $u \leqslant s_{\ell}$ or $u>s_{\ell+1}$. Then

$$
\left[s_{\ell}, s_{\ell+1}\right) \subset\left[t-2^{-j}, t+2^{-j}\right),
$$

and using inequality $(3.2)$ for $j-1$, we see that

$$
\left|x_{s_{\ell+1}}^{m}-x_{s_{\ell}}^{m}\right| \leqslant \frac{2\|F\|_{\gamma}}{1-2^{-\gamma}} 2^{-(j-1) \gamma} .
$$

The number of indices $\ell \in K_{j}$ is at most $2^{j}$. Using the fact that the family $\left(K_{j}\right)_{1 \leqslant j \leqslant m}$ defines a partition of $\{0, \ldots, N-1\}$, and adding inequalities (3.3) and (3.4) for $q>1 / \gamma$, we then have

$$
\begin{aligned}
\sum_{i=0}^{N-1}\left|x_{s_{i+1}}^{m}-x_{s_{i}}^{m}\right|^{q} & \leqslant\left(\frac{2\|F\|_{\gamma} 2^{\gamma}}{1-2^{-\gamma}}\right)^{q} \sum_{j=0}^{m} 2^{j} 2^{-j \gamma q} \\
& \leqslant\left(\frac{2\|F\|_{\gamma} 2^{\gamma}}{1-2^{-\gamma}}\right)^{q} \frac{1}{1-2^{-\gamma q+1}}
\end{aligned}
$$

and we derive

$$
\left\|x^{m}\right\|_{q-\operatorname{var}} \leqslant \frac{2\|F\|_{\gamma} 2^{\gamma}}{1-2^{-\gamma}} \frac{1}{\left(1-2^{-\gamma q+1}\right)^{1 / q}} .
$$

From Helly' selection principle, see e.g. Theorem 6.1 in Chistyakov and Galkin's work [9], the sequence $x^{m}$ has a convergent subsequence in $p$-variation, for any $p>q$. We identify the path $f$ from the statement as such a limit. One proves that $f$ is a selection of $F$ in the same way as we proved that $v_{t} \in F\left(z_{t}\right)$ in Section 2.3 , using the fact that $F(t)$ is closed for all times, and the regularity properties of $F$. 


\section{4 - Rough differential inclusions}

This section is dedicated to the proof of Theorem 1.3 and Theorem 1.4. We refer the reader to Appendix A for basics on rough paths theory. All we need to know is recalled there. The reader will notice that the proof of Theorem 1.3 is much shorter than the proof of Theorem 1.4. This is due to the fact that we somehow assume much more in Theorem 1.3. asking in particular that the set-valued drift has compact convex images. Together with the assumed upper semicontinuity, this allows for the use of powerful approximate selection theorems that greatly simplify the matter. See e.g. Section 1 of Chapter 2 in [1] for the differential inclusion case.

\section{1 - Upper semicontinuous drift}

This section is dedicated to proving Theorem 1.3 , we work in this section in the setting of that statement. Here, $\mathbf{X}$ is an $\alpha$-Hölder weak geometric rough path with $\alpha \in(1 / 3,1 / 2]$ and $G \in C_{b}^{2, \gamma}$ with $\alpha(2+\gamma)>1$. We recall that according to the notation introduced in the Introduction, $C^{1}\left([0, T], \mathbb{R}^{d}\right)$ is the space of $\mathbb{R}^{d}$-valued Lipschitz paths defined on $[0, T]$. Given $x \in C^{1}\left([0, T], \mathbb{R}^{d}\right)$, we denote by $\psi(t, x)$ the solution path to the rough differential equation

$$
y_{t}=\xi+x_{t}+\int_{0}^{t} G\left(y_{s}\right) d \mathbf{X}_{s}
$$

with fixed initial condition $\xi$. The controlled solution path comes under the form of a pair $\left(y_{t}, G\left(y_{t}\right)\right)$. Classical results from rough paths theory ensure that $\psi$ is a continuous function from $C^{1}\left([0, T], \mathbb{R}^{d}\right)$ with values in the space $C^{\alpha}\left([0, T], \mathbb{R}^{d}\right)$ - see e.g. Theorem 3 of [10. This is where we need the assumption that $G$ is $(2+\gamma)$-Hölder, rather than just $(1+\gamma)$-Hölder, as in the proof of Theorem 1.4 given in the next section. For each $0 \leqslant t \leqslant T$, one has

$$
\psi(t, x)=\psi(t, \bar{x}),
$$

for any other $\bar{x} \in C^{1}\left([0, T], \mathbb{R}^{d}\right)$ that coincides with $x$ on the time interval $[0, t]$. We can then work in the setting of differential inclusions with memory - see e.g. Section 7 in Chapter 4 of Aubin \& Cellina's book [1]. Let us define

$$
\mathcal{F}(t, x):=F(t, \psi(t, x)) .
$$

The function $\mathcal{F}$ is upper semicontinuous on $\mathbb{R}_{+} \times C^{1}\left([0, T], \mathbb{R}^{d}\right)$, with values in the set of convex compact subsets of $\mathbb{R}^{d}$. We can then use the obvious variant of Theorem 1 in Section 7 in Chapter 4 in [1, with no constraint and $K(t)=\mathbb{R}^{d}$ for all $t$ with the notations therein, to get the existence of a time $T^{*} \in(0, T]$, and a Lipschitz path $x$, defined on the time interval $\left[0, T^{*}\right]$, such that one has for almost all $t \in\left[0, T^{*}\right]$

$$
\dot{x}_{t} \in \mathcal{F}(t, x) .
$$

This condition is equivalent to saying that the path $y$ from (4.1) solves the rough differential inclusion

$$
d y_{t} \in F\left(t, y_{t}\right)+G\left(y_{t}\right) d \mathbf{X}_{t}
$$

(Note that the convexity assumption on the pointwise images of $F$ is essential for the use Theorem 1 in Section 7 in Chapter 4 of Aubin-Cellina's book [1].) 


\section{2 - Lower semicontinuous drift}

This section is dedicated to proving Theorem 1.4. We take advantage in that task of the approach to Filipov's theorem given by Bressan in [7, based on a selection theorem of independent interest. We recall the setting before embarking on the proof of Theorem 1.4.

Recall also that a cone of $\mathbb{R}^{m}$ is a subset $\Gamma$ of $\mathbb{R}^{m}$ such that

$$
\Gamma \cap(-\Gamma)=\{0\},
$$

and $\lambda a \in \Gamma$, if $\lambda \geqslant 0$ and $a \in \Gamma$. As an example, given a positive constant $M$, the set

$$
\Gamma_{M}:=\left\{(t, x) \in \mathbb{R} \times \mathbb{R}^{d} ; t \geqslant 0,\|x\| \leqslant t M\right\} \subset \mathbb{R}^{d+1}
$$

is a cone of $\mathbb{R}^{d+1}$.

Definition - Let $\Gamma$ be a cone of $\mathbb{R}^{m}$. A map $h: \mathbb{R}^{m} \rightarrow \mathbb{R}^{d}$ is said to be $\Gamma$-continuous at point $a \in \mathbb{R}^{m}$, if for any $\varepsilon>0$, there is $\delta>0$ such that

$$
\|h(b)-h(a)\|<\varepsilon
$$

for any $b \in B(a, \delta) \cap(a+\Gamma)$.

We say that $h$ is $\Gamma$-continuous on a subset $S \subset \mathbb{R}^{m}$, if $h$ is $\Gamma$-continuous at any point of $S$. The relevance of the notion of $\Gamma$-continuity in the setting of differential inclusions is a consequence of the following selection result, due to Bressan, Theorem 1 in [7].

4.1. Theorem - Let $H: \mathbb{R}^{m} \rightarrow \dot{2}^{\mathbb{R}^{d}}$ be lower semicontinuous set-valued map with non-empty closed values. Then, for any cone $\Gamma \subset \mathbb{R}^{m}$, there is a $\Gamma$-continuous selection of $H$.

For a positive finite constant $L$, and $(\beta, \xi) \in(0,1] \times \mathbb{R}^{d}$, set

$$
\mathcal{E}_{L}^{\beta}:=\left\{u \in C^{0}\left([0, T], \mathbb{R}^{d}\right) ;\|u\|_{\beta} \leqslant L, u_{0}=\xi\right\},
$$

where $\|u\|_{\beta}$ is the $\beta$-Hölder norm of $u$, for $0<\beta<1$, and $\|u\|_{1}$ is the Lipschitz norm of $u$. The set $\mathcal{E}_{L}^{\beta}$ is a compact subset of $\left(C^{0}\left([0, T], \mathbb{R}^{d}\right),\|\cdot\|_{\infty}\right)$. We do not emphasize the dependence on $\xi$ and $T$ in the notation.

4.2. Lemma - Assume the set-valued map $F$ is defined on a closed subset $\Omega$ of $\mathbb{R} \times \mathbb{R}^{d}$ where it is lower semicontinuous and takes values in the set $\mathcal{K}\left(\mathbb{R}^{d}\right)$ of nonempty compact subsets of $\mathbb{R}^{d}$. Assume further that there is a positive constant $L$ such that $[0, T] \times B\left(\xi, L T^{\beta}\right) \subset \Omega$, and

$$
\|F(t, x)\| \leqslant L,
$$

for all $(t, x) \in[0, T] \times B\left(\xi, L T^{\beta}\right)$. Then there exists a continuous map

$$
\phi: \mathcal{E}_{L}^{\beta} \rightarrow \mathcal{E}_{L}^{1}
$$

such that one has

$$
\frac{d \phi(u)(t)}{d t} \in F(t, u(t)), \quad \text { for almost all } t \in[0, T]
$$

for any $u \in \mathcal{E}_{L}^{\beta}$.

Proof. We first extend $F$ into a lower semicontinuous map $F^{*}$ defined on $\mathbb{R} \times \mathbb{R}^{d}$, setting

$$
F^{*}(t, x)=B(0, L),
$$


if $(t, x) \notin \Omega$. Pick $M>L$, and use Theorem 4.1 to pick a $\Gamma_{M}$-continuous selection $f$ of $F^{*}$. For $u \in \mathcal{E}_{L}^{\beta}$, and $0 \leqslant t \leqslant T$, set

$$
\phi(u)(t):=\xi+\int_{0}^{t} f\left(s, u_{s}\right) d s .
$$

We have $\left\|f\left(t, u_{t}\right)\right\| \leqslant L$, since $u \in \mathcal{E}_{L}^{\beta}$, and given the assumptions on $F$. So $\phi(u) \in \mathcal{E}_{L}^{1}$, and one has indeed the inclusion 4.2 for almost all times.

One can proceed as follows to show the continuity of the map $\phi$. Since $f$ is $\Gamma_{M^{-}}$ continuous, one can associate to any time $t \in[0, T]$ a positive $\eta_{t}$ such that for all $s \in$ $\left[t, t+\eta_{t}\right] \cap[0, T]$ and $\left\|a-u_{t}\right\| \leqslant M \eta_{t}$, one has

$$
\left\|f(s, a)-f\left(t, u_{t}\right)\right\| \leqslant \frac{\varepsilon}{4 T} .
$$

Write LeB for Lebesgue measure on the real line. From Lemma 1 in Bressan's work [7], there is a finite family $\left(\left[t_{i}, t_{i}+\eta_{t_{i}}^{\frac{1}{\beta}}\right)\right)_{1 \leqslant i \leqslant N}$ of disjoint intervals, such that

$$
\operatorname{LEB}\left(\bigcup_{i=1}^{N}\left[t_{i}, t_{i}+\eta_{t_{i}}^{\frac{1}{\beta}}\right)\right) \geqslant T-\frac{\varepsilon}{8 L} \text {. }
$$

Define

$$
\delta:=\min \left(\left(\frac{\varepsilon}{8 N L}\right)^{\alpha}, \min _{1 \leqslant i \leqslant N} \frac{\eta_{t_{i}}}{2}\right)(M-L)
$$

and set

$$
J_{i}:=\left[t_{i}, t_{i}+\left(\frac{\delta}{M-L}\right)^{\frac{1}{\alpha}}\right)
$$

Then

$$
\operatorname{LEB}\left(\bigcup_{i=1}^{N} J_{i}\right) \leqslant N\left(\frac{\delta}{M-L}\right)^{\frac{1}{\alpha}} \leqslant \frac{\varepsilon}{8 L} \text {. }
$$

Pick now $v \in \mathcal{E}_{L}^{\beta}$ such that $\|u-v\|_{\infty} \leqslant \delta$. For

$$
t \in\left(\bigcup_{i=1}^{N}\left[t_{i}, t_{i}+\eta_{t_{i}}^{\frac{1}{\beta}}\right)\right) \backslash\left(\bigcup_{i=1}^{N} J_{i}\right)=: I \backslash J,
$$

one has $t \in\left[t_{i}+\left(\frac{\delta}{M-L}\right)^{\frac{1}{\alpha}}, t_{i}+\eta_{t_{i}}^{\frac{1}{\alpha}}\right)$, for an index $i$, and

$$
\begin{aligned}
\left\|v(t)-u\left(t_{i}\right)\right\| & \leqslant\|v(t)-u(t)\|+\left\|u(t)-u\left(t_{i}\right)\right\| \\
& \leqslant \delta+L\left(t-t_{i}\right)^{\beta} \\
& \leqslant M\left(t-t_{i}\right)^{\beta}
\end{aligned}
$$

while

$$
\left\|u_{t}-u_{t_{i}}\right\| \leqslant L\left(t-t_{i}\right)^{\beta}<M\left(t-t_{i}\right)^{\beta} .
$$


Thus, with 4.3 we obtain that, for $t \in I \backslash J$, we have

$$
\begin{aligned}
\left\|f\left(t, v_{t}\right)-f\left(t, u_{t}\right)\right\| & \leqslant\left\|f\left(t, v_{t}\right)-f\left(t_{i}, u_{t_{i}}\right)\right\|+\left\|f\left(t_{i}, u_{t_{i}}\right)-f\left(t, u_{t}\right)\right\| \\
& \leqslant \frac{\varepsilon}{2 T} .
\end{aligned}
$$

Since $f$ is bounded by $L$ on $[0, T] \times B\left(z_{0}, L T^{\beta}\right)$, it follows that we have for any $t \in[0, T]$ the estimate

$$
\begin{aligned}
& \|\phi(v)(t)-\phi(u)(t)\| \\
& \leqslant \int_{0}^{T}\left\|f\left(t, v_{s}\right)-f\left(t, u_{s}\right)\right\| d s \\
& \leqslant \int_{I \backslash J}\left\|f\left(s, v_{s}\right)-f\left(s, u_{s}\right)\right\| d s+\int_{J \cup[0, T] \backslash I}\left\|f\left(s, v_{s}\right)-f\left(t, u_{s}\right)\right\| d s \\
& \leqslant T \frac{\varepsilon}{2 T}+(\operatorname{LEB}(J)+\operatorname{LEB}([0, T] \backslash I)) 2 L \\
& \leqslant \frac{\varepsilon}{2}+\left(\frac{\varepsilon}{8 L}+\frac{\varepsilon}{8 L}\right) 2 L \leqslant \varepsilon .
\end{aligned}
$$

We use from now on the notations on controlled paths recalled in Appendix A. Recall the one form $G$ in Theorem 1.4 is assumed to be $C_{b}^{1, \gamma}$, with $1<\alpha(2+\gamma)<3 \alpha$. Choose a constant $\beta \in(1 / 3, \alpha)$ such that $\beta(2+\gamma)>1$.

We define a ball in the space of paths controlled by $X \in C^{\alpha}\left([0, T], \mathbb{R}^{\ell}\right)$, using the $\beta$-norm

$$
B_{L}^{\beta}:=\left\{\left(y, y^{\prime}\right) \in \mathcal{D}^{\beta, 2 \beta}\left([0, T], \mathbb{R}^{d}\right) ;\left\|\left(y, y^{\prime}\right)\right\|_{\beta, 2 \beta} \leqslant L, y_{0}=\xi, y_{0}^{\prime}=G(\xi)\right\} .
$$

It follows from Ascoli-Arzela theorem that this ball is a compact convex subset of

$$
C^{0}\left([0, T], \mathbb{R}^{d}\right) \times C^{0}\left([0, T], L\left(\mathbb{R}^{\ell}, \mathbb{R}^{d}\right)\right),
$$

with both factor endowed with the uniform norm. For $\left(y, y^{\prime}\right) \in \mathcal{D}^{\beta, 2 \beta}\left([0, T], \mathbb{R}^{d}\right)$, and $t \in[0, T]$, we set

$$
\Phi\left(y, y^{\prime}\right)(t):=\left(\phi(y)(t)+\int_{0}^{t} G\left(y_{s}\right) d \mathbf{X}_{s}, G\left(y_{t}\right)\right) .
$$

We prove below that $\Phi$ is a continuous map from $B_{L}^{\beta}$ into itself, provided $T$ is small enough. See point (a) for the stability and point (b) for the continuity. It follows then from Schauder fixed point theorem that $\Phi$ has a fixed point $\left(\tilde{z}, \tilde{z}^{\prime}\right)$ in $B_{L}^{\beta}$. One gets the fact that $\left(\tilde{z}, \tilde{z}^{\prime}\right) \in \mathcal{D}^{\alpha, 2 \alpha}\left([0, T], \mathbb{R}^{d}\right)$ from the equation that it satisfies, as (A.3) yields the estimate

$$
\begin{aligned}
\|\tilde{z}\|_{\alpha} \leqslant & L T^{1-\alpha}+\|G(\tilde{z})\|_{\infty}\|X\|_{\alpha}+\left\|G(\tilde{z})^{\prime}\right\|_{\infty}\|\mathbb{X}\|_{2 \alpha} \\
& +C\|\mathbf{X}\|_{\beta}\left\|\left(G(\tilde{z}), G(\tilde{z})^{\prime}\right)\right\|_{\beta,(1+\gamma) \beta} T^{(2+\gamma) \beta-\alpha},
\end{aligned}
$$

from which we get $\left\|\tilde{z}^{\prime}\right\|_{\alpha} \leqslant\|G\|_{C_{b}^{1}}\|z\|_{\alpha}$, and finally for $R_{s t}^{\tilde{z}}:=\tilde{z}_{s t}-\tilde{z}_{s}^{\prime} X_{s t}$

$$
\begin{aligned}
\left\|R^{\tilde{z}}\right\|_{2 \alpha} \leqslant & L T^{1-2 \alpha}+\left\|G(\tilde{z})^{\prime}\right\|_{\infty}\|\mathbb{X}\|_{2 \alpha} \\
& +C\|\mathbf{X}\|_{\beta}\left\|\left(G(\tilde{z}), G(\tilde{z})^{\prime}\right)\right\|_{\beta,(1+\gamma) \beta} T^{(2+\gamma) \beta-2 \alpha} .
\end{aligned}
$$

The triple $\left(\left(\tilde{z}, \tilde{z}^{\prime}\right), \phi(\tilde{z})\right)$ is then a solution of the rough differential inclusion 1.5 . 
(a) Stability - Write $R_{s t}^{y}$ for $y_{s t}-y_{s}^{\prime} X_{s t}$, for any $0 \leqslant s \leqslant t \leqslant T$, and similary for $z$ where $\left(z, z^{\prime}\right)=\Phi\left(y, y^{\prime}\right)$. On the one hand, for $\left(y, y^{\prime}\right) \in B_{L}^{\beta}$, one has

$$
\begin{aligned}
\|y\|_{\beta} & \leqslant\left\|y^{\prime}\right\|_{\infty}\|X\|_{\beta}+\left\|R^{y}\right\|_{2 \beta} T^{\beta} \\
& \leqslant\left(\left\|y_{0}^{\prime}\right\|+\left\|y^{\prime}\right\|_{\beta} T^{\beta}\right)\|X\|_{\beta}+\left\|R^{y}\right\|_{2 \beta} T^{\beta} \\
& \leqslant\left(\|G(\xi)\|+L T^{\beta}\right) T^{\alpha-\beta}\|X\|_{\alpha}+L T^{\beta} .
\end{aligned}
$$

We then have $y \in \mathcal{E}_{L}^{\beta}$. On another hand, according to Proposition A.3. one has

$$
\left(G(y), G(y)^{\prime}\right) \in \mathcal{D}^{\beta, \beta(\gamma+1)},
$$

because $\beta \gamma<2 \beta$. Using $\mathrm{A.3}$ and the fact that $\|\mathbb{X}\|_{2 \beta} \leqslant T^{\alpha-\beta}\|\mathbb{X}\|_{2 \alpha}$, one has for any $s, t \in[0, T]$,

$$
\begin{aligned}
\left\|R_{s t}^{z}\right\| & =\left\|z_{s t}-z_{s}^{\prime} X_{s t}\right\| \\
& \leqslant\left\|\phi(y)_{s t}\right\|+\left\|\int_{s}^{t} G\left(y_{r}\right) d \mathbf{X}_{r}-G\left(y_{s}\right) X_{s t}\right\| \\
& \leqslant L|t-s|+\left\|G(y)^{\prime}\right\|_{\infty}\|\mathbf{X}\|_{2 \beta}|t-s|^{2 \beta} \\
& +C\|\mathbf{X}\|_{\beta}\left\|\left(G(y), G(y)^{\prime}\right)\right\|_{\beta, \beta(\gamma+1)}|t-s|^{\beta(2+\gamma)} \\
& \leqslant\left(L T^{1-2 \beta}+\left\|G(y)^{\prime}\right\|_{\infty}\|\mathbb{X}\|_{2 \alpha} T^{\alpha-\beta}\right. \\
& \left.+C\|\mathbf{X}\|_{\beta}\left\|\left(G(y), G(y)^{\prime}\right)\right\|_{\beta, \beta(\gamma+1)} T^{\beta \gamma}\right)|t-s|^{2 \beta} .
\end{aligned}
$$

Using the estimate of the size of the nonlinear image of a controlled path given in A.2, one has eventually for $\left\|R^{z}\right\|_{2 \beta}$ the upper bound

$$
\begin{aligned}
& \left(L T^{1-2 \beta}+\|G\|_{C_{b}^{1,0}}\|y\|_{\infty}\|\mathbb{X}\|_{\beta} T^{\alpha-\beta}+C\|\mathbf{X}\|_{\alpha}\|G\|_{C_{b}^{1, \gamma}}(1+\|G(\xi)\|+L)^{2} T^{\beta \gamma}\right) \\
& \leqslant T^{\alpha-\beta}\left(L+\|G\|_{C_{b}^{1,0}}\|y\|_{\infty}\|\mathbb{X}\|_{\beta}+C\|\mathbf{X}\|_{\alpha}\|G\|_{C_{b}^{1, \gamma}}(1+\|G(\xi)\|+L)^{2}\right)
\end{aligned}
$$

where we use the inequalities $T^{\alpha-\beta} \geqslant T^{1-2 \beta}, T^{\beta \gamma}$ in the last line. Since

$$
\left\|R^{y}\right\|_{2 \beta} \leqslant L
$$

we also have

$$
\|y\|_{\infty} \leqslant\|\xi\|+\|G(\xi)\|\|X\|_{\beta} T^{\beta}+L T^{2 \beta} .
$$

Hence, we obtain from (4.6) and (4.7) the upper bound

$$
\begin{aligned}
& T^{\alpha-\beta}\left(L+\|\sigma\|_{C_{b}^{1,0}}\|\mathbb{X}\|_{\beta}\left(\|\xi\|+\|G(\xi)\|\|X\|_{\beta} T^{\beta}+L T^{2 \beta}\right)\right. \\
& \left.+C\|\mathbf{X}\|_{\alpha}\|G\|_{C_{b}^{\gamma-1}}(1+\|G(\xi)\|+L)^{2}\right)
\end{aligned}
$$


for $\left\|R^{z}\right\|_{2 \beta}$. We control the $\beta$-Hölder norm of $z^{\prime}$ as follows

$$
\begin{aligned}
\left\|z^{\prime}\right\|_{\beta} & \leqslant\|G\|_{C_{b}^{1,0}}\|y\|_{\beta} \\
& \leqslant\|G\|_{C_{b}^{1,0}}\left(\left\|y^{\prime}\right\|_{\infty}\|X\|_{\beta}+\left\|R^{y}\right\|_{2 \beta} T^{\beta}\right) \\
& \leqslant\|G\|_{C_{b}^{1,0}}\left(\left(\left\|y_{0}^{\prime}\right\|+\left\|y^{\prime}\right\|_{\beta} T^{\beta}\right)\|X\|_{\alpha} T^{\alpha-\beta}+\left\|R^{y}\right\|_{2 \beta} T^{\beta}\right) \\
& \leqslant\|G\|_{C_{b}^{1,0}}\left(\left(\|G(\xi)\|+L T^{\beta}\right)\|X\|_{\alpha} T^{\alpha-\beta}+L T^{\beta}\right) \\
& \leqslant\|G\|_{C_{b}^{1,0}}\left(\left(\|G(\xi)\|+L T^{\beta}\right)\|X\|_{\alpha}+L\right) T^{\alpha-\beta}
\end{aligned}
$$

With $T$ small enough, we obtain from (4.8) and 4.9) that $\left\|\left(z, z^{\prime}\right)\right\|_{\alpha, 2 \alpha} \leqslant L$. This shows indeed that $\Phi$ sends $B_{L}^{\beta}$ into itself.

(b) Continuity - The continuity of $\Phi$ on $B_{L}^{\beta}$, for the uniform topology on $B_{L}^{\beta}$, is a direct consequence of the continuity of $\phi$ and the continuity estimate A.3 on rough integrals.

\section{A - Basics on Young and rough integrals}

Let $p \geqslant 1$ be given. Recall that an $\mathbb{R}^{d}$-valued path $x$ defined on the time interval $[a, b]$. We say that $x$ has finite $p$-variation over that interval $[s, t] \subset[a, b]$ if

$$
\|y\|_{p-\operatorname{var},[s, t]}^{p}:=\sup \sum_{i}\left|y_{s_{i+1}}-y_{s_{i}}\right|^{p}<\infty
$$

where the supremum runs over the set of finite partitions $\left\{s_{i}\right\}$ of the interval $[s, t]$. We denote by $V_{p}\left([a, b], \mathbb{R}^{d}\right)$ the set of $\mathbb{R}^{d}$-valued path with finite $p$-variation over $[a, b]$ and by $C^{1 / p}\left([a, b], \mathbb{R}^{d}\right)$ the set of $\mathbb{R}^{d}$-valued path $\frac{1}{p}$-Hölder continuous on $[a, b]$. Note that an element of $V_{p}\left([a, b], \mathbb{R}^{d}\right)$ need not be continuous, while $C^{1 / p}\left([a, b] \mathbb{R}^{d}\right) \subset V_{p}\left([a, b], \mathbb{R}^{d}\right)$. Also, a continuous path with finite $p$-variation has a reparametrized version that is $\frac{1}{p}$-Hölder over its interval of definition. Refer to [9] for a reference. We endow the space of paths with finite $p$-variation with the norm

$$
\|y\|_{p \text {-var, } \infty,[a, b]}:=\|y\|_{p \text {-var, }[a, b]}+\|y\|_{\infty,[a, b]},
$$

where $\|\cdot\|_{\infty,[a, b]}$ denotes the uniform norm over $[a, b]$. Similarly, for $\alpha \in[0,1]$, we define a norm on the space of $\mathbb{R}^{d}$-valued $\alpha$-Hölder functions defined on the interval $[a, b]$, setting

$$
\|x\|_{\alpha, \infty,[a, b]}:=\|x\|_{\alpha,[a, b]}+\|x\|_{\infty},
$$

where $\|\cdot\|_{\alpha,[a, b]}$ is the classical semi-norm of $\alpha$-Hölder paths. For these norms and seminorms, we omit to precise the interval $[a, b]$ when the norms and semi-norms are taken over the whole interval of the path is defined.

Given $a \leqslant b$, set $\Delta_{a, b}:=\{(s, t) ; a \leqslant s \leqslant t \leqslant b\}$. A control over the interval $[a, b]$ is a map $\omega: \Delta_{a, b} \mapsto \mathbb{R}^{+}$that is null on the diagonal, is non-increasing, resp. non-decreasing, as a function of each of its first, resp. second, argument, and is sub-additive

$$
\omega(s, u)+\omega(u, t) \leqslant \omega(s, t), \quad \forall a \leqslant s \leqslant u \leqslant t \leqslant b .
$$

Denote by $\omega\left(s, t^{-}\right)$the left limit in $t$ of the non-decreasing function $\omega(s, \cdot)$. A control is said to be regular if it is continuous in a neighbourhood of the diagonal. As an example, for any path $y \in V_{p}\left([a, b], \mathbb{R}^{d}\right)$, the function $\omega_{y}(s, t):=\|y\|_{p \text {-var, }[s, t]}^{1 / p}$ is a control. For an 
$\mathbb{R}^{d}$-valued $\alpha$-Hölder path $x$, with $0<\alpha<1$, the function $\omega_{x}(s, t):=\|x\|_{\alpha,[s, t]}^{1 / \alpha}$ is a regular control.

From the present day perspective, Gubinelli' sewing lemma [16] offers an easy road to constructing the Young integral - see also [13]. We give here a variation due to Friz and Zhang [15], tailor-made to our needs. Given a map $\mu: \Delta_{a, b} \mapsto \mathbb{R}^{d}$ and a finite partition $\pi=\left\{s_{i}\right\}$ of the interval $[a, b]$, set

$$
\mu_{\pi}:=\sum_{i} \mu_{s_{i+1} s_{i}}
$$

A.1. Proposition - Let a map $\mu: \Delta_{a, b} \mapsto \mathbb{R}^{d}$ be given. If there exists positive exponents $\alpha_{1}, \alpha_{2}$, with $\alpha_{1}+\alpha_{2}>1$ and controls $\omega_{1}, \omega_{2}$, with

$$
\left|\mu_{t s}-\left(\mu_{t u}+\mu_{u s}\right)\right| \leqslant \omega_{1}(s, u)^{\alpha_{1}} \omega_{2}(u, t)^{\alpha_{2}}, \quad \forall a \leqslant s \leqslant u \leqslant t \leqslant b,
$$

with $\omega_{2}$ regular, then, for any interval $[s, t] \subset[a, b]$, the $\mu_{\pi_{s t}}$ converge to some limit $I_{s}^{t}(\mu)$ as the mesh of the partition $\pi_{t s}$ of $[s, t]$ tends to 0 , and one has

$$
\left|I_{s}^{t}(\mu)-\mu_{\pi_{s t}}\right| \leqslant C \omega_{1}\left(s, t^{-}\right)^{\alpha_{1}} \omega_{2}(s, t)^{\alpha_{2}},
$$

for some positive constant $C$ depending only on $\alpha_{1}, \alpha_{2}$.

Given $x \in C^{\alpha}\left([a, b], \mathbb{R}^{\ell}\right)$ and $y \in V_{p}\left([a, b], L\left(\mathbb{R}^{\ell}, \mathbb{R}^{d}\right)\right)$, set

$$
\mu_{s t}:=y_{s}\left(x_{t}-x_{s}\right) \text {, }
$$

and note that

$$
\mu_{t s}-\left(\mu_{t u}+\mu_{u s}\right)=\left(y_{s}-y_{u}\right)\left(x_{t}-x_{u}\right), \quad s \leqslant u \leqslant t,
$$

so one has for any interval $[s, t]$ the estimate

$$
\left|\mu_{t s}-\left(\mu_{t u}+\mu_{u s}\right)\right| \leqslant\|y\|_{p \text {-var, }[s, t]}^{1 / p}\|x\|_{\alpha,[s, t]}^{\alpha} .
$$

A.2. Corollary - If $\frac{1}{q}+\alpha>1$, the Riemann sums $\mu_{\pi_{s t}}$ associated with the preceding two-index map $\mu$ converge to some limit which we denote by $\int_{s}^{t} y_{u} d x_{u}$. One has

$$
\left\|\int_{0} y_{u} d x_{u}\right\|_{\alpha,[s, t]} \lesssim\|y\|_{q-v a r,[s, t]}\|x\|_{\alpha,[s, t]},
$$

and for any $\varepsilon>0$ with $\frac{1-\varepsilon}{q}+\alpha>1$, and $y, y^{\prime} \in V_{q}\left([a, b], L\left(\mathbb{R}^{\ell}, \mathbb{R}^{d}\right)\right)$, one has

$$
\begin{aligned}
\left\|\int_{0} y_{u} d x_{u}-\int_{0} y_{u}^{\prime} d x_{u}\right\|_{\alpha,[s, t]} & \lesssim\left\|y-y^{\prime}\right\|_{\infty,[s, t]}^{\varepsilon} \\
& \times\left(\|y\|_{q-\operatorname{var}, \infty,[s, t]}^{1-\varepsilon}+\left\|y^{\prime}\right\|_{q-\operatorname{var}, \infty,[s, t]}^{1-\varepsilon}\right)\|x\|_{\alpha,[s, t]} .
\end{aligned}
$$

We refer the reader to the lecture notes [8, 17, 6] for pedagogical accounts of rough paths theory. The following definitions and propositions will be sufficient for our needs here.

Definition - Let $\alpha \in(1 / 3,1 / 2)$. We say that $\mathbf{X}:=(X, \mathbb{X})$ is an $\alpha$-Hölder rough path if

- $X$ is an $\mathbb{R}^{\ell}$-valued $\alpha$-Hölder path on a time interval $[0, T]$,

- $\mathbb{X}$ is a path from $[0, T]^{2}$ to $\mathbb{R}^{\ell} \otimes \mathbb{R}^{\ell}$ such that

$$
\|\mathbb{X}\|_{2 \alpha}:=\sup _{s, t \in[0, T], s \neq t} \frac{\left\|\mathbb{K}_{s t}\right\|}{|t-s|^{2 \alpha}}<+\infty,
$$


- we have

$$
\mathbb{K}_{r t}-\mathbb{X}_{r s}-\mathfrak{X}_{s t}=X_{r s} \otimes X_{s t}
$$

for all $0 \leqslant r \leqslant s \leqslant t \leqslant T$.

The formula

$$
\|\mathbf{X}\|_{\alpha}:=\|X\|_{\alpha}+\|\mathbb{X}\|_{2 \alpha}
$$

defines a seminorm on the space of $\alpha$-Hölder rough paths.

Definition - Let $\alpha \in(0,1], \theta>\alpha$ and $X \in C^{\alpha}\left([0, T], \mathbb{R}^{\ell}\right)$. A path

$$
y \in C^{\alpha}\left([0, T], \mathbb{R}^{d}\right)
$$

is said controlled by $X$, with a remainder of order $\theta$, if there is a path

$$
y^{\prime} \in C^{\theta-\alpha}\left([0, T], L\left(\mathbb{R}^{\ell}, \mathbb{R}^{d}\right)\right),
$$

and a map $R:[0, T]^{2} \rightarrow \mathbb{R}^{d}$, such that

$$
\|R\|_{\theta}:=\sup _{s, t \in[0, T], t \neq s} \frac{\left\|R_{s, t}^{y}\right\|}{|t-s|^{\theta}}<\infty .
$$

and

$$
y_{s t}=y_{s}^{\prime} X_{s t}+R_{s t} .
$$

We denote by $\mathcal{D}^{\alpha, \theta}\left([0, T], \mathbb{R}^{d}\right)$ the space of such pairs $\left(y, y^{\prime}\right)$, and talk of $\left(y, y^{\prime}\right)$ as a controlled path. We make no reference to the reference path $X$ in the notation as there is no risk of confusion. We define a semi-norm on $\mathcal{D}^{\alpha, \theta}\left([0, T], \mathbb{R}^{d}\right)$ setting

$$
\left\|\left(y, y^{\prime}\right)\right\|_{\alpha, \theta}:=\left\|y^{\prime}\right\|_{\theta-\alpha}+\left\|R^{y}\right\|_{\theta}
$$

a norm is defined by the formula

$$
\left\|\left(y, y^{\prime}\right)\right\|_{\alpha, \theta}^{*}:=\left\|y_{0}\right\|+\left\|y_{0}^{\prime}\right\|+\left\|\left(y, y^{\prime}\right)\right\|_{\alpha, \theta} .
$$

The next proposition says that the class of controlled paths is stable by nonlinear maps. See Corollary 3 and Proposition 4 of Gubinelli's original statement [16].

A.3. Proposition - For $\left(y, y^{\prime}\right) \in \mathcal{D}^{\alpha, \theta}\left([0, T], \mathbb{R}^{d}\right)$, and $f \in C_{b}^{1, \varepsilon}$ with $\varepsilon \in(0,1]$, one has

$$
\left(f(y), f(y)^{\prime}\right):=\left(f(y), D f(y) y^{\prime}\right) \in \mathcal{D}^{\alpha, \theta^{\prime}}
$$

with $\theta^{\prime}:=\min (\theta, \alpha(1+\varepsilon))$, and

$$
\begin{aligned}
& \left\|\left(f(y), f(y)^{\prime}\right)\right\|_{\alpha, \theta^{\prime}} \lesssim_{T}\|f\|_{C_{b}^{1, \varepsilon}} \\
& \quad\left(\left\|y_{0}^{\prime}\right\|+\left\|\left(y, y^{\prime}\right)\right\|_{\alpha, \theta}+\left[\left\|y_{0}^{\prime}\right\|+\left\|\left(y, y^{\prime}\right)\right\|_{\alpha, \theta}\right]^{1+\varepsilon}+\left[\left\|y_{0}^{\prime}\right\|+\left\|\left(y, y^{\prime}\right)\right\|_{\alpha, \theta}\right]^{\theta^{\prime} / \alpha}\right),
\end{aligned}
$$

for an implicit multiplicative constant depending only on $T$, that decreases to 0 when $T$ goes to 0.

For a controlled path $\left(y, y^{\prime}\right) \in \mathcal{D}^{\alpha, \theta}\left([0, T], \mathbb{R}^{d}\right)$, with $\alpha+\theta>1$, it is elementary to see that the two-index map

$$
\mu_{s t}:=y_{s} X_{s t}+y_{s}^{\prime} \bigvee_{s t},
$$

satisfies the estimate

$$
\left|\mu_{s t}-\mu_{s u}-\mu_{u t}\right| \lesssim|t-s|^{\alpha+\theta},
$$


for all $0 \leqslant s \leqslant u \leqslant t \leqslant T$, so one can use the sewing lemma to make sense of the integral

$$
\int_{0} y_{s} d \mathbf{X}_{s}
$$

as the additive functional associated to $\mu$. It satisfies, for any $s, t \in[0, T]$, the estimate

$$
\left\|\int_{s}^{t} y_{r} d \mathbf{X}_{r}-y_{s} X_{s t}-y_{s}^{\prime} \mathcal{K}_{s t}\right\| \lesssim\|\mathbf{X}\|_{\alpha}\left\|\left(y, y^{\prime}\right)\right\|_{\alpha, \theta}|t-s|^{\alpha+\theta}
$$

\section{Acknowledgement}

The second author of this article thanks the Center for Mathematical Modeling, Conicyt fund AFB 170001.

\section{References}

[1] Aubin, J.-P. and Cellina, A. Differential inclusions. Set-valued maps and viability theory. Springer, 1984.

[2] Aubin, J.-P. and da Prato, G., Stochastic Viability and Invariance. Annali Scuola Normale di Pisa, 27:595-694, 1990.

[3] Aubin, J.-P. and da Prato, G., Stochastic Nagumo's viability theorem. Stoch. Anal. Appl., 13(1):111, 1995.

[4] Aubin, J.-P. and da Prato, G., The Viability Theorem for Stochastic Differential Inclusions. Stochastic Anal. Appl., 16(1):1-15, 1998.

[5] Aubin, J.-P. and da Prato, G. and Frankowska, H., Stochastic invariance for differential inclusions. Set-valued Anal., 8:181-201, 2000.

[6] Bailleul, I., A flow-based approach to rough differential equations. https://perso.univrennes1.fr/ismael.bailleul/files/M2Course.pdf, 2015.

[7] Bressan, Alberto, Directionally continuous selections and differential inclusions, Funkcial. Ekvac, 31(3):459-470, 1988.

[8] Caruana, M. and Lévy, Th. and Lyons, T.J., Differential Equations Driven by Rough Paths. Lect. Notes Math., 1908, 2007.

[9] Chistyakov, V.V and Galkin, O.E., On maps of bounded $p$-variation with $p>1$. Positivity, 2:19-45, 1998.

[10] Coutin, L. and Lejay, A., Sensitivity of rough differential equations: an approach through the Omega lemma, Journal of Differential Equations, 264(6): 3899-3917 , 2018

[11] Coutin, L. and Marie, N., Invariance for rough differential equations. Sto. Proc. Appl., 127(7):23732395, 2017.

[12] Davie, A. M., Differential equations driven by rough paths: an approach via discrete approximation. Appl. Math. Res. Express. AMRX, 2007.

[13] Feyel, D. and de la Pradelle, A., Curvilinear integrals along enriched paths. Elec. J. Probab., 11(34):860-892, 2006.

[14] Friz, P. and Gess, B. and Gulisashvili, A. and Riedel, S., The Jain-Monrad criterion for rough paths and applications to random Fourier series and non-Markovian Hörmander theory. Ann. Probab. 44(1):684-738, 2016.

[15] Friz, P. and Zhang, H., Differential equations driven by rough paths with jumps. J. Diff. Eq., 264(10):6226-6301, 2018.

[16] Gubinelli, M., Controlling rough paths. J. Funct. Anal., 216:86-140, 2004.

[17] Friz, P. and Hairer, M., A course on rough paths. Springer, 2014.

[18] Kisielewicz, M. Differential inclusions and optimal control. Mathematics and its Applications (East European Series), 44, Kluwer Academic Publishers Group, Dordrecht; PWN-Polish Scientific Publishers, Warsaw, 1991.

[19] Kisielewicz, M. Strong and weak solutions to stochastic inclusions. Banach Center Publ., 32:277286, Geometry in nonlinear control and differential inclusions (Warsaw, 1993), Polish Acad. Sci. Inst. Math., Warsaw, 1995. 
[20] Kisielewicz, M Stochastic differential inclusions and applications. Springer Optimization and Its Applications, 80, Springer, New York, 2013.

[21] Lejay, A., Controlled Differential Equations as Young Integrals: A Simple Approach. J. Diff. Eq., 248:1777-1798, 2010.

[22] Levakov, A. A. and Vas'kovskii, M. M. Existence of solutions of stochastic differential inclusions with standard and fractional Brownian motions. Translation of Differ. Uravn. - Differ. Equ., 51(8):991-997, 2015.

[23] Lyons, T., Differential equations driven by rough signals. I. An extension of an inequality of L. C. Young. Math. Res. Lett., 1(4):451-464, 1991.

[24] Lyons, T.J., Differential equations driven by rough signals. Rev. Mat. Iberoamericana, 14(2):215$310,1998$.

[25] Mandelbrot, B. and Van Ness, J.W., Fractional Brownian motions, fractional noises and applications. SIAM Rev.,10:422-437, 1968.

[26] Young, L. C., An inequality of Hölder type, connected with Stieltjes integration. Acta Math., 67:251-282, 1936.

- I. Bailleul - Institut de recherche mathématiques de Rennes, CNRS UMR 6625, Université de Rennes, 263 Avenue du General Leclerc 35042 RENNES, France.

email: ismael.bailleul@univ-rennes1.fr

- A. Brault - MAP5, CNRS UMR 8145, Université de Paris, Paris, France, and Center for Mathematical Modeling, UMI CNRS 2807, University of Chile, Chile.

email: abrault@dim.uchile.cl

- L. Coutin - Institut de Mathématiques de Toulouse, CNRS UMR 5219, Université Paul Sabatier, 118 Route de Narbonne, 31062 Toulouse Cedex 9, France.

email: laure.coutin@math.univ-toulouse.fr 